\title{
Amabilidad digital frente a nuevas generaciones. Perfil digital del periodista madrileño y su relación con el principiante
}

\author{
Alexandra María Sandulescu Budea \\ Universidad Rey Juan Carlos, España \\ alexandra.sandulescu@uric.es
}

Recibido: 24/2/2018 / Aceptado: 15/3/2018

doi: 10.26439/contratexto2018.n029.1821

\begin{abstract}
Resumen. El presente artículo monitoriza la presencia y gestión del perfil digital en medios sociales por parte de periodistas madrileños y el grado de identificación con las nuevas generaciones que siguen sus cuentas. Para ello, partimos de una selección de perfiles digitales en función de los índices de rendimiento en publicación. El objetivo es describir cómo las diferentes generaciones de periodistas en Madrid utilizan los recursos disponibles en las plataformas digitales, siendo capaces de generar interacción frente a un seguidor que quiere trabajar en el mismo campo (un estudiante). Para llevar a cabo este proceso, la investigación contará con una fase de documentación, una de testeo y otra de aplicación e interpretación de resultados; el proceso completo abarcó desde agosto del 2017 a enero del 2018. La metodología combina variables cuantitativas y cualitativas. Las conclusiones revelan que hay un desfase en los perfiles a medida que se amplía el ciclo generacional del periodista.
\end{abstract}

Palabras clave: periodismo digital / medios sociales / ciberperiodismo / análisis de usuarios / participación 


\title{
Digital Kindness for New Generations. Madrilenian Journalists' Digital Profile and its Relationship with Beginners
}

\begin{abstract}
This article monitors the presence and management of the digital profile in social media by Madrilenian journalists, and the degree of identification with the new generations that follow their accounts. We started our research by selecting digital profiles based on performance indexes in publications. We aim to describe how the different generations of journalists in Madrid make use of the resources available on digital platforms, thus being able to generate interaction with followers who want to work on the same field (students). To carry out this process, the research had three phases: documentation, testing, and application and interpretation of results. The complete process took place from August 2017 to January 2018. The methodology combined quantitative and qualitative variables. Finally, the research revealed that there is a gap between the profiles, as the generational cycle of journalists increases.

Keywords: digital journalism / social media / cyberjournalism / user analysis / participation
\end{abstract}




\section{Introducción}

$\mathrm{M}$ uchos de los informes consultados establecen que la digitalización del periodismo no es una realidad, sino que trasciende, se especializa y se vuelve consumible; es decir, se mediatiza de tal forma que empieza a mezclar producto y consumo (Fundación Telefónica, 2017). De esta forma, encontramos que prácticamente el $64 \%$ de los jóvenes de todo el mundo entre 18 y 24 años usan las redes sociales como fuente informativa (Vara-Miguel, Negredo y Amoedo, 2017), mientras que en España el tráfico de datos para la obtención de fuentes fiables se centra en la consulta de un consumo de información estandarizado en el perfil del usuario que lanza dicha información (Fundación Telefónica, 2017).

De esta forma, las nuevas generaciones, sin darse cuenta, imponen su uso como consumo mediático (EFE, 2017) diversificando y especializando las plataformas. Cuando hablamos del grado de identificación con la figura del periodista y la posibilidad por parte de las nuevas generaciones de acceder a dichos mercados, la forma de visualización más clara queda anclada en cómo se percibe digitalmente la profesión a partir de la gestión de los propios perfiles profesionales. Este aspecto está íntimamente relacionado con la práctica profesional, la confianza o credibilidad que genera frente a sus seguidores, así como con la gestión reputacional de su propia marca de autor importada del mundo offline (Newman, Levy y Nielsen, 2015). Esa imagen que se proyecta a través de los medios sociales es más personal que profesional (Asociación de la Prensa de Madrid, 2016), con lo que apunta a elevados porcentajes de uso en plataformas donde los propios periodistas reconocen que el promedio de contacto corresponde a una franja muy específica de entre 18 y 24 años (Kirk, Mc Namara, Culloty y Suiter, 2016). Así, aunque la temática no siempre esté relacionada con el ejercicio de la profesión, su revisión es continua, ya que es una forma de detectar tendencias que se pueden manifestar en opiniones encontradas en las propias redes sociales (Asociación de la Prensa de Madrid, 2016).

Desde este punto de vista, la educación de los jóvenes universitarios en las ramas de periodismo en España pasa por enseñar cómo se deben gestionar tales perfiles en la identificación del periodista profesional, de manera que pueda reencontrar su legitimidad como actor social y gestionar su propia presencia digital en las redes sociales, en paralelo a la producción de contenidos. Esto implica diseñar estrategias de gestión con la participación de audiencias activas, así como valorar las consecuencias de una publicación, una comunicación o un comentario como espacio de interacción periodística y gestión de la propia reputación. Para ello, se debe verificar, generar confianza y saber en todo momento el lenguaje que hay que utilizar (Falla, 2011).

Para López García y Campos Freire (2015), las redes sociales han demostrado que son capaces de alterar la producción informativa y la agenda de 
los medios más tradicionales al hacer circular la información de una manera antes impensable. Este fenómeno, sin embargo, no sería posible si el propio periodista no fuera capaz de generar esa interactividad con el usuario, marcada por comentarios, reacciones o difusiones en otras plataformas sociales, de manera que consigue "verificar casi en tiempo real el interés que suscita un tema" (Rost, 2012, p. 3).

En este sentido, proponemos la idea de observar tanto las fluctuaciones de los perfiles digitales como las consecuencias reales que producen un contacto efectivo. Un usuario medio consulta con facilidad una media de cuatro redes sociales diariamente (We Are Social, 2016), y no suele utilizar una plataforma social de forma exclusiva. Para Rodríguez (2017), de entre las más conocidas en España, Twitter suele usarse para informarse (10 \%), Facebook para relacionarse (44\%) y YouTube para entretenerse (19\%). Sin embargo, las plataformas se diversifican en función de las generaciones, por lo que se puede añadir WhatsApp (8\%), Instagram (3\%) o LinkedIn en proporciones residuales (EFE, 2017). De este modo, el tránsito de la universidad al mundo laboral pasa por entrenar el desarrollo del criterio en una realidad cada vez más tecnificada, desestructurada e híbrida como parte integrante del futuro periodista.

Salaverría (2005) considera que esto supone no solo analizar y jerarquizar la información, sino también tomar un papel activo que impregna la realidad social con una opinión digital a la manera de la teoría del agenda-setting (Wolf, 1991) y con una actualización constante del mensaje que organiza las estructuras mentales en la actualidad. En el ámbito digital, esto se traduce en un posicionamiento con una segmentación ordenada en torno a objetivos muy concretos, que generan una atención específica hacia unas visualizaciones que limitan la búsqueda de nuevos parámetros (Davenport y Beck, 2001). Todos ellos son propios del ecosistema digital (Herrero, 2015) y pueden dar lugar a nuevas tendencias en cuanto a cómo se presenta un periodista en la esfera pública (Sandulescu, 2017). La percepción de esos perfiles profesionales anclados digitalmente obliga a aplicar métodos de investigación concretos para la difusión de competencias y la formación de futuros periodistas.

Hoy en día, un perfil digital puede mostrar lo que somos y cómo somos, a través de un contenido concreto (Rodríguez, 2017). Los profesionales de la información lo usan como herramienta de trabajo e instrumento de construcción de una identidad digital propia, marcando diferencias en la proyección y consolidación de profesionales (Yuste y Cabrera, 2014). Aquello se traduce en su capacidad para construir un perfil específico que opera al margen de su actividad profesional, pero que integra dinámicas que luego utilizará, como generar una red de contactos confiable a la cual recurrir, contribuir a la construcción de su credibilidad y aportar valor. Por lo tanto, ello se traduce en tres aspectos clave: el tratamiento de 
hechos o acontecimientos, la actualidad y la periodicidad como fundamentos de la profesión periodística (Gago, 2007). Todos ellos se encuentran dentro del equilibrio de realidad, veracidad y actualidad, propios de la producción informativa (Fontcuberta, 1993).

El perfil del periodista pasa, entonces, de la polivalencia a la especialización progresiva de las redes sociales, donde su trabajo no solo es la búsqueda de fuentes y la promoción de sus artículos, sino la identificación y escucha de una audiencia específica. Esta complementa la cobertura de temas, recoge sugerencias, críticas, testimonios, opiniones e impresiones agregadas a las redacciones, que impactan en el desarrollo de la agenda diaria (Sandulescu, 2017). Es lo que ya en su momento Negroponte (1995) reconocía como la transformación de la completa estructura mental hacia la digitalización, donde las destrezas profesionales se muestran por el grado de identificación obtenido en su presencia virtual.

En esta línea, la situación del periodista es consecuencia de una evolución progresiva producto de lo que Hernández (2012) denomina pasos sound (p. 1). Esto se refiere a la adaptación progresiva del soporte, que no solo ha modificado estructuras culturales y avances tecnológicos, sino también las formas de pensar y de presentarnos ante la sociedad. Asimismo, constituye un modo de "espectacularización del yo" (Sibilia, 2008, p. 2), que se presenta como un dosier laboral donde valores, pensamientos y formas de actuar complementan un perfil. Su objetivo es conquistar la visibilidad mostrando subjetivamente la actividad en una constante exposición que retroalimenta la propia actividad. Para el profesional de la información, esto supone un nivel de competitividad alto, que pueda superar otras formas disponibles en los entornos digitales. Si el contenido no recibe la suficiente participación, se incentiva atendiendo a valoraciones, opiniones y actividades de usuarios.

Las características esenciales en el perfil del periodista del siglo xxI son, por tanto, el autoaprendizaje continuo en nuevas herramientas y la especialización en las estrategias comunicativas de las redes sociales (López García y Campos Freire, 2015). Lewis, Molton y Coddington (2014) lo categorizan a través de tres tipos de participación que los periodistas pueden tener con sus seguidores:

1. Participación directa o conversación mediante intercambio directo de mensajes.

2. Participación indirecta o formas de actuación en beneficio de la comunidad.

3. Participación sostenida, manifestada en la actividad de páginas o plataformas sociales que mantienen la colaboración e interacción de forma duradera.

Desde este punto de vista, entendemos la necesidad de conocer no solo el grado de integración entre plataformas, sino la comunicación que generan las publicaciones con contenido de calidad, diferente y bien contextualizado, que otorguen credibilidad y confianza; cómo se produce esa 
gestión del contenido y cómo se comunica con su público. No solo en el ámbito del comentario, sino, si existiera, una identificación frente a un perfil descendente, es decir, monitorizar una reputación con un seguimiento digital para poder analizar, de forma puntual y regular en el tiempo, el clima de opinión. Estos elementos son estudiados en torno a un personaje específico, que es el periodista, y el consejo que pueden dar a una futura generación que quiere dedicarse a esa profesión (Rodríguez, 2017). El enfoque u objeto formal se sitúa en la evolución que se produce en la sectorialización de perfiles, su gestión mediática y el grado de respuesta frente a situaciones planteadas a ítems específicos.

\section{Metodología}

Para disponer de un informe serio, es preciso detenerse a analizar e investigar la actividad digital del profesional a lo largo de un período amplio. Esto incluye no solo publicaciones sociales, sino cualquier manifestación en el entorno digital. Se formulan, entonces, las primeras preguntas en torno a los siguientes aspectos:

1. ¿Qué monitorizar?, conociendo tanto valores positivos como negativos sobre parámetros determinados. Acotar características del entorno, crear listas con frases para localizar opiniones, posibles fallos, competidores contextuales, referencias e índices de actividad.

2. ¿Dónde monitorizar?, estableciendo búsquedas filtradas con prioridad en los perfiles personales públicos, y adaptadas hacia figuras públicas notificadas como tal en las plataformas que indican correctamente las descripciones específicas.

3. ¿Cómo monitorizar?, buscando por palabras o términos, observando actividades y rastreando temáticas especiales, según necesidades concretas.

4. ¿Para qué monitorizar? Así, se puede obtener una información y un análisis que reflejará los datos necesarios para analizar todo lo observado e interpretarlo.

La metodología de estudio es propia de la disciplina métrica digital en el área del análisis en redes sociales, anclada en las ciencias de la comunicación. La hipótesis principal que plantea esta investigación es que las redes sociales deberían servir para fomentar la comunicación útil y que, si un seguidor hace una pregunta - dado su perfil público y su función como periodista-, este debería responderla. Así, la investigación se planteó en tres fases: exploratoria, preparatoria y de actuación concreta.

\section{Fase exploratoria}

Según la Asociación de la Prensa de Madrid (2016, 2017), en el 2018 se encuentran en España 1756 profesionales que son reconocidos por tres características: están federados, realizan una actividad continua en los medios de comunicación y poseen un título acreditado. Bajo este 
argumento de autoridad, seleccionamos una muestra inicial de 1000 periodistas que cumplieran con los requisitos de ser profesionales con actividad digital y presencia en redes sociales.

Esta muestra se redujo a 500 cuando nos centramos en redes sociales específicas (Facebook, Twitter, Instagram y LinkedIn), y se restringió una vez más a 300 cuando seleccionamos aquellos que tuvieran un perfil con un índice de publicación regular. Esta se mide por directrices que da cada plataforma para la obtención de una rentabilidad mínima, ya sea esta económica, de atención o conversión (Rodríguez, 2017). El período de esta fase de la investigación va desde agosto a septiembre del 2017. El proceso es complejo en cuanto implica la revisión unitaria de cada uno de los candidatos para descubrir su viabilidad.

Del mismo modo, las propias redes sociales, para ser efectivas, deben tener una frecuencia computable en su publicación. Según datos de Social Bakers (2018), el promedio en Facebook es la actualización diaria con dos publicaciones mínimas, entre tres y cinco tuits por día en Twitter, una publicación semanal en LinkedIn y al menos una publicación cada dos o tres días en Instagram (Villegas, 2017). Esto se debe hacer atendiendo siempre a la geolocalización de las zonas horarias y al comportamiento específico de cada plataforma, tomando como modelo los estudios publicados en el 2016 de Nelio'.

Figura 1. Métricas sociales

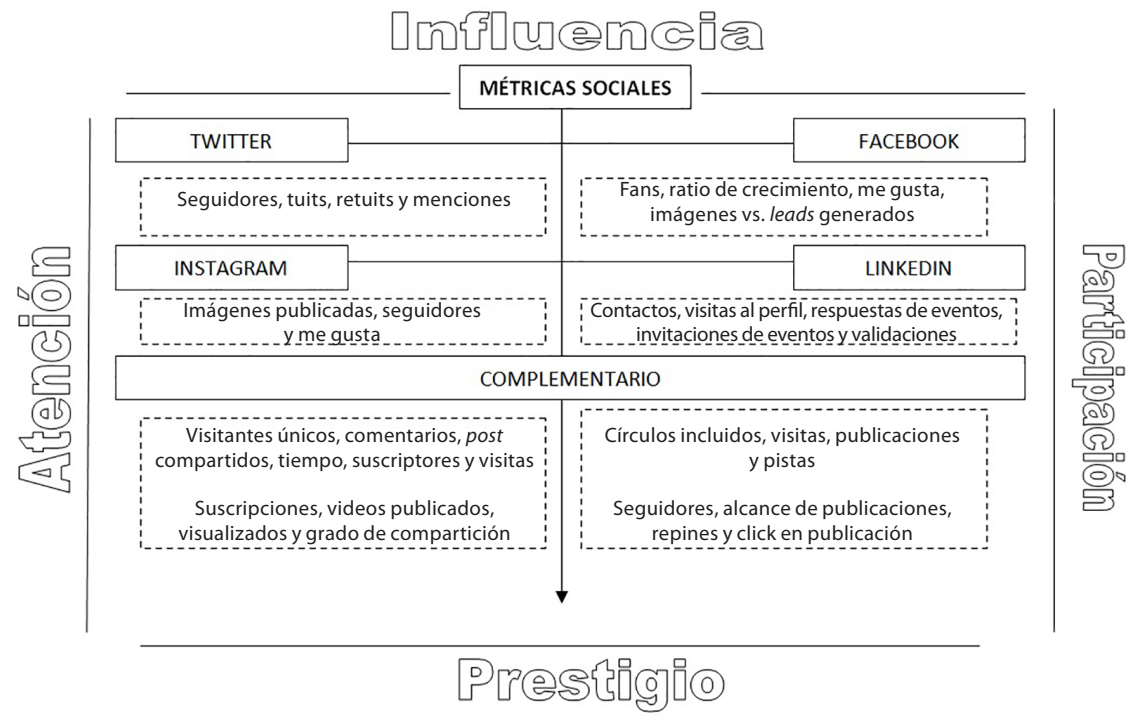

1 Esta investigación contempla también la red social Pinterest. 
Figura 2. Franjas de publicación en redes sociales

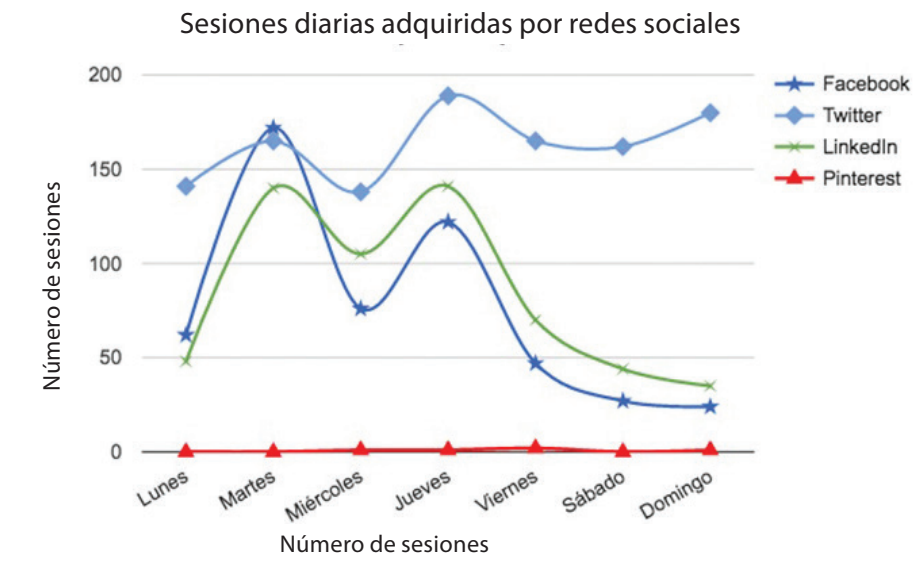

Fuente: Nelio. Equipo de desarrollo (2016)

Para establecer la geolocalización de las zonas horarias de cada una de las plataformas sociales, recurrimos a los informes de segmentación precisa de datos demográficos y segmentación automática ofrecidos por cada compañía ${ }^{2}$. Esto produjo la disminución de la muestra en 210 periodistas.

En cuanto a aspectos concretos relacionados con la muestra, diferenciamos tres grupos de edad: aquellos nacidos entre 1950-1970, entre 1970-1980 y entre 1980-2000. Para los participantes nacidos antes de 1950, no encontramos perfiles porque no cumplían con las características mínimas de estar activos.
En el caso de los nacidos después del 2000, los perfiles eran de estudiantes. Partiendo del ideario de generación digital (Fundación Telefónica, 2017), este coincide con las clasificaciones establecidas. Con respecto al porcentaje de uso en redes sociales o medios, se observan bastantes diferencias (véase la figura 3).

Las generaciones más antiguas no usan Instagram, frente a las más jóvenes (lo que se demuestra también en su perfil de trabajo); en cambio, los jóvenes no terminan de ver la utilidad de LinkedIn. Twitter o Facebook se encuentran bastante estables tanto en generaciones como en profesiones.

2 La información se encuentra contenida en el área de cada plataforma. 
Figura 3. Especificaciones de la muestra

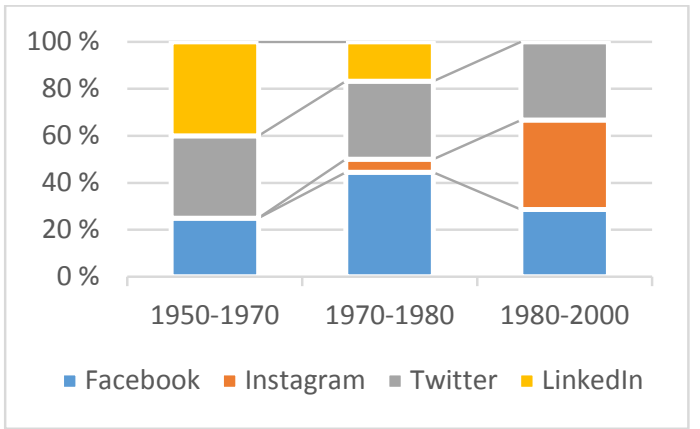

Elaboración propia

\section{Fase preparatoria}

Una vez seleccionada la muestra final, se procedió a realizar un estudio unitario de cada uno de los perfiles y su índice de actividad para cada plataforma entre los meses de octubre y noviembre del 2017. No todos los periodistas tenían actividad en todas las plataformas, por lo que se tuvo que hacer la observación de uno en uno para monitorizar la presencia digital. Para ello, aplicamos herramientas específicas de recopilación de búsquedas como Monitter (para

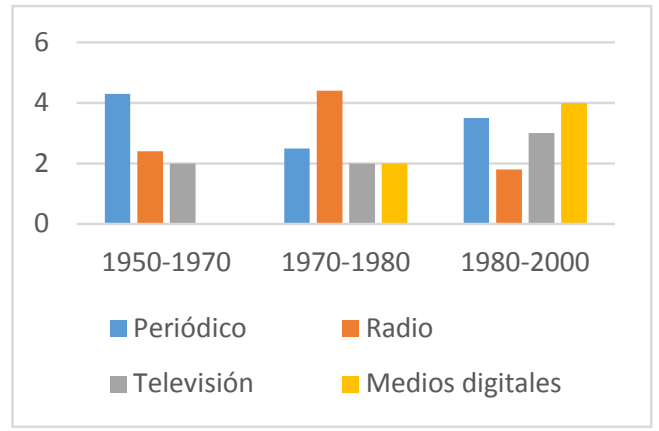

actividades relacionadas con Twitter), Social Mention (a través de resultados de palabras clave), Radian6 (para el seguimiento de todas las conversaciones que suceden en tiempo real) y Socialmetrix (para marcar un entorno equilibrado entre análisis cuantitativo y cualitativo), que relacionamos con década generacional y actividad a través de la fecha de nacimiento e información pública. Esto nos permitió saber si existía efectividad a la hora de poner en marcha la siguiente fase.

Figura 4. Monitorización de reputación del perfil por plataforma

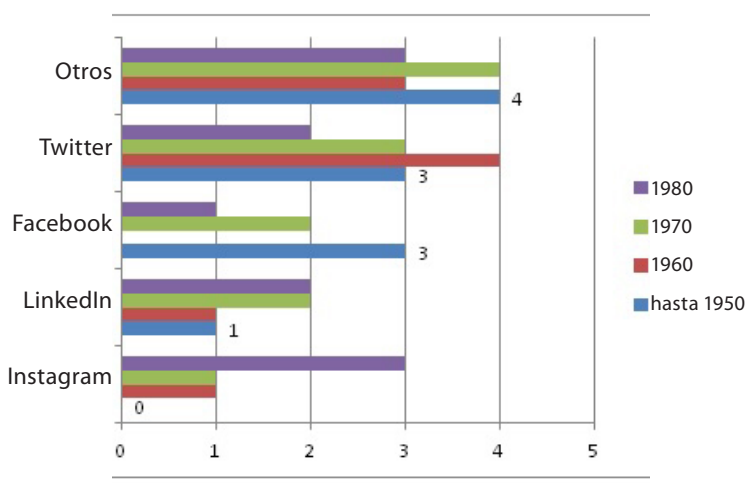


Los datos fueron agrupados según dos perspectivas:

a. Década generacional en la que nació el periodista, que se divide en cuatro áreas: aquellos nacidos hasta la década de 1950, durante los años 1960, en los años 1970 y en la década de 1980. No se contempló la década de 1990 porque los periodistas seleccionados formaban parte de proporciones muy residuales ${ }^{3} \mathrm{y}$, por tanto, se afinaron los criterios de análisis especializándose por década.

b. Efectividad de uso por plataforma, que se mide estableciendo un baremo entre 1 y 5 , siendo 1 poco utilizado y 5 muy utilizado.

Los resultados obtenidos nos enseñaron qué estrategias teníamos que emplear: para aquellos periodistas que habían nacido en 1950, lo más efectivo era mandar un correo o ponerse en contacto con la redacción en la que trabajaran, ya que existían pocas probabilidades en redes sociales.

Con aquellos que habían nacido en 1960, existía un 90 \% de probabilidades de que contestaran a través de Twitter, ya que Facebook se reservaba para el entorno privado, mientras que tanto Instagram como LinkedIn eran utilizados de forma residual. Sin embargo, con respecto al total de todas las décadas, esta generación complementaba Twitter con correos institucionales: la gran parte de los perfiles mostraba direcciones de correo como información adicional.

Para aquellos nacidos entre los años 1970 y 1980, las principales diferencias se encontraban en el uso de Instagram (hay mayor actividad en generaciones más jóvenes) y en la forma de contacto: aquellos nacidos en los años setenta prefieren terminar el contacto presencialmente, mientras que los nacidos en los años ochenta optan por el WhatsApp o la conversación telefónica.

La monitorización nos permitió averiguar el grado de fidelidad e identificación entre la plataforma y el periodista. Las generaciones más antiguas preferían una presencia en pocas plataformas con mayor desarrollo de contenido, a diferencia de las generaciones más nuevas, que tenían presencia en todas las plataformas, pero duplicando y copiando contenido entre ellas.

Por otro lado, la mención del apartado "otros" incluye procesos complementarios a la plataforma, como correos institucionales o páginas web del periodista. Aquí llama la atención que las generaciones de 1950 y 1970 presenten valores idénticos con respecto al uso de páginas web, frente a las de 1980 y 1960, que tienen valores idénticos en cuanto a la utilización de un correo o número de WhatsApp para la puesta en contacto.

3 De los 1756 profesionales, tres cumplían con todas las características y uno pasó todo el proceso de análisis, pero tras seis solicitudes de contacto sin respuesta se optó por no continuar. 
Estos datos fueron muy valiosos para esta investigación, ya que con ellos por fin podíamos aplicar nuestro verdadero objetivo: averiguar si se podría producir una verdadera sinergia entre el profesional y el estudiante. Esta fase terminó de desarrollarse en diciembre del 2017.

\section{Fase de actuación concreta}

Una vez localizada la muestra final, identificados qué periodistas usaban más cada plataforma y seleccionadas las categorías de análisis, procedimos a buscar una muestra aleatoria de alumnos universitarios. Estos fueron convocados por correo electrónico y contaban con los siguientes requisitos:

- Alumnos universitarios que cursaran Periodismo o dobles grados que lo combinaran.

- Alumnos con posibilidad de reunión física en Madrid en la Universidad Rey Juan Carlos.

- Alumnos que estuvieran dispuestos a contactar personalmente a los periodistas, según las directrices del coordinador.

- Alumnos que tuvieran una actividad diaria comprobable en al menos dos de las plataformas que son objeto de estudio.

De una muestra inicial de 100 alumnos, seleccionamos 70. Cada alumno recogió de una urna al azar tres periodistas y, siguiendo las indicaciones del coordinador, realizó una monitorización simple de visitante, usuario y contenido en torno a tres conceptos: opinión de la profesión periodística, consejos del profesional y grado de adaptabilidad con los nuevos soportes. Con la información obtenida durante el mes de enero del 2018, se solicitaba un consejo al propio periodista, a la vez que se le informaba de todo el proceso.

Con ello, lo que pretendimos fue, por un lado, que el alumno fuera consciente de que las redes sociales en el campo periodístico son instrumentos de trabajo no solo para la generación de contenidos, sino también para la creación de relaciones, y, por otro lado, descubrir el grado de identificación de un usuario-periodista con respecto a sus seguidores. Esto significa conocer cómo reaccionan cuando alguien quiere ser como tú y te pide un consejo.

\section{Resultados}

Lo más interesante de esta investigación es, sin lugar a dudas, las dos vertientes contrapuestas con las que nos hemos encontrado. Por un lado, hemos obtenido un índice reputacional de identidad alto, que ha generado incluso reuniones presenciales. Por otro lado, hemos visto cómo otros ignoraron completamente cualquier acercamiento mientras continuaban con su actividad digital.

Durante el mes de enero del 2018, se llevaron a cabo dos fases. En la primera, se procedió a establecer el contacto con cada uno de los profesionales, según el modelo estándar suministrado por el coordinador: presentación amigable, nombre del interesado, explicación del 
proyecto y solicitud de ayuda o consejo. El proceso comprendía hasta tres intentos por cada una de las plataformas y vías de contacto (página web, correo o teléfono) dadas por el coordinador para cada uno de los tres periodistas asignados a cada alumno. La segunda fase consistió en la realización de la entrevista.

De los 210 periodistas asignados, 67 llegaron al final del proceso ${ }^{4}$. Las causas de las bajas estuvieron relacionadas con no responder a las solicitudes (60 usuarios), problemas de agenda e incompatibilidad de horarios (50 usuarios), enfermedad o accidente (10 usuarios) o negativa del propio usuario (23 usuarios). Aquellos que no pudieron responder por problemas de agenda o incompatibilidad de horarios alegaron razones como la presentación de un libro (20 usuarios), trabajo en dos sitios (12 usuarios), viaje fuera de España (8 usuarios), motivos de trabajo (6 usuarios), embarazo y maternidad (4 usuarios). Los 67 periodistas que finalizaron el proceso arrojaron resultados muy interesantes y valiosos para la profesión periodística ante las siguientes preguntas:

1. ¿El periodismo es vocacional? El $95 \%$ respondió afirmativamente (solo hubo tres personas que no estuvieron de acuerdo). Lo consideran como un requisito indispensable para poder sobrevivir en la profesión.

2. ¿Merece la pena ser periodista en España? De 67, 10 respondieron un no rotundo; sin embargo, asociado a la vocación, la gran mayoría argumentó que, aun siendo una profesión complicada, otorga gran satisfacción.

3. ¿Es importante la especialización en el periodismo? 25 periodistas dijeron que no, aun cuando lo relacionaron con una posibilidad para las nuevas generaciones.

4. ¿En qué medida las nuevas tecnologías ayudan al periodismo? En este sentido, las respuestas fueron muy parecidas a la primera pregunta. Prácticamente el $95 \%$ está a favor.

Con respecto al consejo, se pidió a los periodistas que eligiera como mucho dos de los siguientes ítems: curiosidad, trabajo, aguante, fe (destino) o humildad. En la figura 5, podemos ver cómo la capacidad de trabajar es el elemento más valorado y, curiosamente, la humildad es el menos valorado.

4 Por la propia protección de datos personales y solicitud de alguno de los interesados, no se muestran los nombres. Si algún investigador, a título personal, desea más información, puede ponerse en contacto con la autora o consultar las actas del III Congreso Internacional de Comunicación y Pensamiento, que se celebró en Sevilla del 21 al 23 de marzo del 2018, donde se amplían cuestiones específicas relativas a la primera parte de la investigación. 
Figura 5. El consejo del profesional

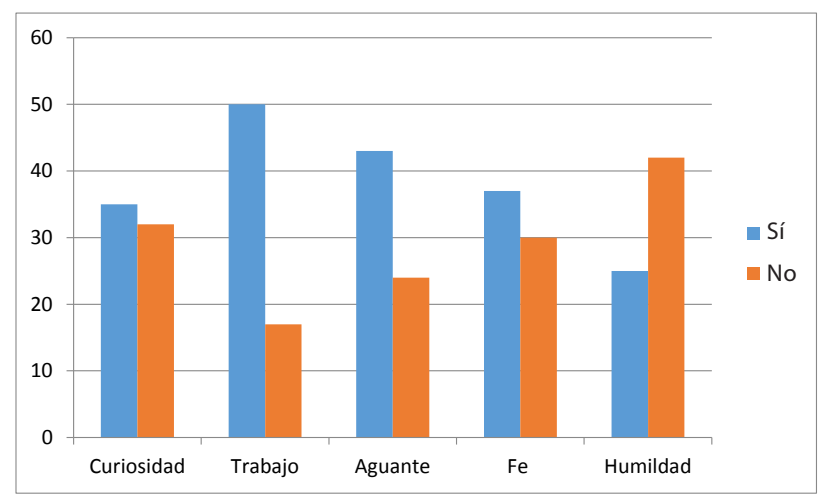

Elaboración propia

Con respecto a la plataforma con mayor índice de comunicación, llama la atención el apartado "otros". Si bien es cierto que Twitter sigue siendo la que tiene mayor índice de respuesta, al final, los periodistas que acceden a responder las preguntas utilizan otros medios. De los 67 entrevistados, 36 optaron por otras vías: la gran mayoría, por el correo electrónico (32), pero también por el teléfono (2) o el WhatsApp (2). Del mismo modo, 50 de los 67 entrevistados se reunieron presencialmente con el alumno invitándolo a participar en el propio desarrollo del programa o actividad periodística.

Figura 6. Índice de respuesta en plataformas

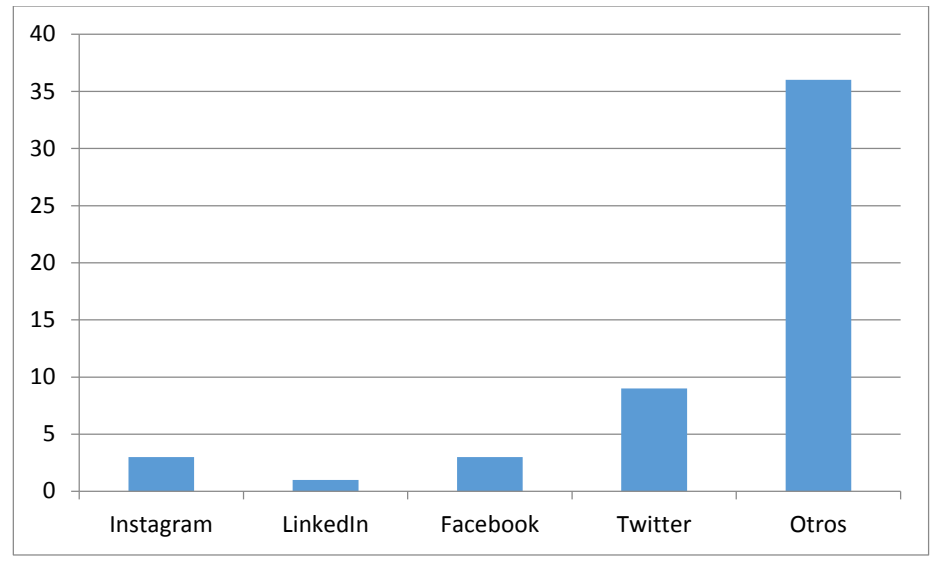

Elaboración propia 


\section{Discusión}

Un aspecto que nos ha llamado la atención en esta investigación ha sido una curiosa asociación que se hacía más evidente conforme íbamos recopilando los datos, coordinando la actividad docente y recolectando información a consecuencia del análisis de interpretación. Durante el desarrollo de esta investigación hemos observado una diferencia en el tono de los mensajes de respuesta bastante importante de unas generaciones con respecto a otras.

Prácticamente el $90 \%$ de los periodistas nacidos hasta 1970 (y los 42 que terminaron el proceso) fueron muy amables, cordiales e incluso invitaron a participar en futuros proyectos a los alumnos. Menos dos de ellos, todos los que no participaron se encontraban en el apartado de problemas de agenda. Aquellos nacidos en la década de 1970 son los que más se resistieron en el contacto, pero al final sí accedieron a participar (23 terminaron el proceso completo). Aquellos que no participaron, no lo hicieron porque no respondieron. Finalmente, lo que nos llamó la atención fue el $100 \%$ de las respuestas de la generación nacida en los años 1990 fueron absolutas: solo dos participaron en el proceso completo y los demás manifestaron negativas con tonos que, en algunas ocasiones, perjudicaban su propia reputación digital.
Hemos querido destacar este aspecto por la preocupación ante la perspectiva de que lo puntual pueda derivar en general. Hay que tener siempre una perspectiva de mentalidad abierta en la idea de que las nuevas generaciones no son un peligro, sino una oportunidad para crear aspectos vitales en una sociedad que debe renovarse si quiere evolucionar.

Los periodistas son modelos de referencia - aun no queriendo serlo- de futuras generaciones que tienen un deseo aspiracional que puede (o no) cumplirse. Nuestro cometido como buenos profesionales es ayudar, en la medida de lo posible, a plantear un escenario para que ellos puedan desarrollarse en un futuro, continuar el legado que les dejemos y asegurar así también nuestra tranquila retirada.

Así, esta investigación nos ha revelado la importancia del acercamiento entre la universidad y los entornos laborales, sobre todo en ámbitos donde los propios profesionales no otorgan una credibilidad absoluta en la metodología docente y la ven como algo separado de la propia profesión. Es posible y deseable preparar al futuro profesional, enseñarle a observar la realidad y mostrarle que esos datos son reales y aportan consecuencias concretas que afectan a su propia vida y desarrollo personal. 


\section{Referencias}

Asociación de la Prensa de Madrid. (2016). Informe anual de la profesión periodística. Recuperado de http://www.apmadrid.es/wp-content/uploads/2017/10/ Informe_anual_profesion_APM_2016_baja_7mg.pdf

Asociación de la Prensa de Madrid. (2017). Informe anual de la profesión periodística. Recuperado de http://corresponsalesdepaz.es/archivos/201712/InformeAPM -2017_2.pdf

Davenport, T., y Beck, J. (Eds.). (2001). The Attention Economy: Understanding the New Currency of Business. Nueva York, NY: Harvard Business School Press.

EFE. (10 de junio del 2017). Periodismo del siglo xxI, una profesión digital. Vanguardia. Recuperado de https://www.vanguardia.com.mx/articulo/periodismo-del-siglo-xxi-una-profesion-digital

Falla, S. (7 de abril del 2011). El perfil del periodista y comunicador digital. Recuperado de http://www.maestrosdelweb.com/perfil-del-periodista-y-comunicador-digital/

Fontcuberta, M. (1993). La noticia. Pistas para percibir el mundo. Barcelona: Paidós.

Fundación Telefónica. (2017). Sociedad digital en España 2017. Barcelona: Editorial Ariel. Recuperado de https://www.fundaciontelefonica.com/arte_cultura/publicaciones-listado/pagina-item-publicaciones/itempubli/625/

Gago, M. (2007). Flash 2.0. Tecnología y cibermedios en la nueva web social. En A. Larrondo y A. Serrano (Eds.), Diseño periodístico en internet (pp. 103-128). Bilbao: Universidad del País Vasco.

Hernández, M. (2012). Pasos sound. Revista Latinoamericana Musical, 3.

Herrero, E. (2015). The Credibility of Social Media in Journalism. Transinformaçao, 27(2), 165-171. Recuperado de http://ref.scielo.org/2m2fwb

Kirk, N., McNamara, P., Culloty, E., y Suiter, J. (2016). Digital News Report Ireland 2016. Reuters Institute for the Study of Journalism. Recuperado de http://fujomedia. eu/wp-content/uploads/2016/06/Digital-News-Report-Ireland-2016.pdf

Lewis, S., Molton, D., y Coddington, M. (2014). Reciprocal Journalism. Journalism Practice, 8(2), 229-241. doi:10.1080/17512786.2013.859840

López García, X., y Campos Freire, F. (Eds.). (2015). Periodismo en cambio. Oporto: Media XXI.

Negroponte, N. (1995). Being Digital. Nueva York, NY: Alfred A. Knopf.

Nelio. Equipo de desarrollo. (2016). Informes. Recuperado de https://neliosoftware. com/es/?s=informes\&submit 
Newman, N., Levy, D. A., y Nielsen, R. K. (2015). Digital News Report 2015. Reuters Institute for the Study of Journalism. Recuperado de http:/ reutersinstitute.politics.ox.ac.uk/sites/default/files/research/files/ Reuters\%2520Institute\%2520Digital\%2520News\%2520Report\%25202015_ Full\%2520Report.pdf

Rezab, J. (11 de abril del 2011). How often should you post on your Facebook pages [mensaje en un blog]. Recuperado de https://www.socialbakers.com/blog/147how-often-should-you-post-on-your-facebook-pages

Rodríguez, Ó. (2017). Curso de community manager. Barcelona: Anaya Multimedia.

Rost, A. (2012). Modelos de uso y gestión de redes sociales en el periodismo. Conferencia presentada en el $V$ Congreso Internacional de Ciberperiodismo y Web 2.0, 12-14 de noviembre. Bilbao. Recuperado de https://www.researchgate.net/ publication/253651025_Modelos_de_uso_y_gestion_de_redes_sociales_en_ el_Periodismo

Salaverría, R. (2005). Redacción periodística en internet. Pamplona: Eunsa.

Sandulescu, A. (2017). Fundamentos de métrica digital en ciencias de la comunicación. Barcelona: UOC.

Sibilia, P. (2008). La intimidad como espectáculo. Buenos Aires: Fondo de Cultura Económica.

Social Bakers. (2018). Studies. Recuperado de https://www.socialbakers.com/socialmedia-content/studies/

Vara-Miguel, A., Negredo, S., y Amoedo, A. (2017). DigitalNewsReport.es.2017 Noticias en manos de la audiencia. Recuperado de http://www.digitalnewsreport.es/

Villegas, A. (2 de mayo del 2017). Frecuencia de publicación óptima en redes sociales [mensaje en un blog]. Recuperado de https://neliosoftware.com/es/blog/ frecuencia-de-publicacion-redes-sociales/

We Are Social. (2016). Digital in 2016. Recuperado de https://www.slideshare.net/wearesocialsg/digital-in-2016/

Wolf, M. (1991). Efectos sociales de los medios de comunicación de masas. Barcelona: Pòrtic.

Yuste, B., y Cabrera, M. (2014). Emprender en periodismo. Nuevas oportunidades para el profesional de la información. Barcelona: UOC. 